\title{
Research priorities to address the global burden of chronic obstructive pulmonary disease (COPD) in the next decade
}

Davies Adeloye ${ }^{1}$, Dhiraj Agarwal ${ }^{2}$, Peter J Barnes ${ }^{3}$, Marcel Bonay ${ }^{4}$, Job F van Boven ${ }^{5}$, Jamie Bryant ${ }^{6}$, Gaetano Caramori ${ }^{7}$, David Dockrell ${ }^{8}$, Anthony D'Urzo ${ }^{9}$, Magnus Ekström ${ }^{10}$, Gregory Erhabor ${ }^{11}$, Cristóbal Esteban $^{12}$, Catherine M Greene ${ }^{13}$, John Hurst ${ }^{14}$, Sanjay Juvekar ${ }^{2}$, Ee Ming Khoo ${ }^{15}$, Fanny W Ko ${ }^{16}$, Brian Lipworth $^{17}$, Jose L López-Campos ${ }^{18}$, Matthew Maddocks ${ }^{19}$, David M Mannino $^{20}$, Fernando J Martinez ${ }^{21}$, Miguel A Martinez-Garcia ${ }^{22}$, Renae J McNamara ${ }^{23}$, Marc Miravitlles ${ }^{24}$, Hilary Pinnock ${ }^{1}$, Alison Pooler ${ }^{25}$, Jennifer K Quint $^{3}$, Peter Schwarz ${ }^{26}$, George M Slavich $^{27}$, Peige Song ${ }^{28}$, Andrew Tai ${ }^{29}$, Henrik Watz ${ }^{30}$, Jadwiga A Wedzicha ${ }^{3}$, Michelle C Williams ${ }^{31}$, Harry Campbell ${ }^{1}$, Aziz Sheikh ${ }^{1}$ Igor Rudan ${ }^{1}$

Affiliations of authors are listed at the end of the manuscript.

\section{Correspondence to:}

Professor Igor Rudan, FRSE Centre for Global Health, Usher Institute The University of Edinburgh 30 West Richmond Street Edinburgh, EH8 9DX, UK. Igor.Rudan@ed.ac.uk
Background The global prevalence of chronic obstructive pulmonary disease (COPD) has increased markedly in recent decades. Given the scarcity of resources available to address global health challenges and respiratory medicine being relatively under-invested in, it is important to define research priorities for COPD globally. In this paper, we aim to identify a ranked set of COPD research priorities that need to be addressed in the next 10 years to substantially reduce the global impact of COPD.

Methods We adapted the Child Health and Nutrition Research Initiative (CHNRI) methodology to identify global COPD research priorities.

Results 62 experts contributed 230 research ideas, which were scored by 34 researchers according to six pre-defined criteria: answerability, effectiveness, feasibility, deliverability, burden reduction, and equity. The top-ranked research priority was the need for new effective strategies to support smoking cessation. Of the top 20 overall research priorities, six were focused on feasible and cost-effective pulmonary rehabilitation delivery and access, particularly in primary/community care and low-resource settings. Three of the top 10 overall priorities called for research on improved screening and accurate diagnostic methods for COPD in low-resource primary care settings. Further ideas that drew support involved a better understanding of risk factors for COPD, development of effective training programmes for health workers and physicians in low resource settings, and evaluation of novel interventions to encourage physical activity.

Conclusions The experts agreed that the most pressing feasible research questions to address in the next decade for COPD reduction were on prevention, diagnosis and rehabilitation of COPD, especially in low resource settings. The largest gains should be expected in low- and middle-income countries (LMIC) settings, as the large majority of COPD deaths occur in those settings. Research priorities identified by this systematic international process should inform and motivate policymakers, funders, and researchers to support and conduct research to reduce the global burden of COPD.

It is estimated that between 300 and 400 million people globally live with chronic obstructive pulmonary disease (COPD) $[1,2]$. The growing burden of COPD is particularly concerning in low- and middle-income countries (LMICs), due to in- 
creasing rates of smoking, household- and ambient air pollution and other exposures, coupled with large and ageing populations [3-5]. Furthermore, the ongoing COVID-19 pandemic highlighted COPD as a condition that predisposes to increased risk of hospitalisation and death [6]. Given the scarcity of resources available for addressing global health challenges and respiratory medicine being relatively underinvested in, it is important to define research priorities for COPD globally [7-9].

COPD now ranks as the fourth leading cause of death, resulting in around three million deaths each year [8,10-12]. Globally, COPD is estimated to result in economic costs of US\$2.1 trillion, and at least half of these costs are now in LMICs [13]. Of this total, an estimated US\$1.9 trillion are direct costs such as medical care, while US $\$ 0.2$ trillion are indirect costs such as missed work [14]. These figures are expected to more than double by the year 2030 [13]. Therefore, a coordinated global response is needed to effectively address the burden of COPD and the very considerable challenges it poses. Prioritising research on COPD could help to motivate leaders, researchers and stakeholders. As resources in global health are generally limited, there is a need to agree on research priorities to guide policymakers and funding organisations as they work to advance the COPD research agenda $[15,16]$.

In response to the heightened interest of the international community in non-communicable diseases, several efforts have been successfully conducted in the past decade to identify research priorities in various fields of global health [17-19]. These initiatives have assisted progress because the process of identification of research priorities has informed governments, funding agencies and the private sector on how to prioritise investments in a systematic way. There was a need for a transparent, systematic and replicable prioritisation process that would be perceived as globally representative and fair. It should involve all relevant stakeholders to mount a coordinated international response to the existing complex and sizable challenges in the field of COPD research. The Child Health and Nutrition Research Initiative (CHNRI) method, now the most commonly used methodological approach to generate research priorities for medicine and health care, was developed to respond to this need for methodological rigour. It has been successfully used as a tool to assist decision making and consensus development in child health and nutrition [20-22] and subsequently been extended to numerous other priority-setting exercises [23], including those in research on disability [24], dementia [25], global mental health [26] and medication safety [27].

Past research prioritisation exercises conducted in the field of COPD have been insightful, but have used a variety of approaches and methods that fell short of being global, systematic or replicable outside of the specific context, or were limited in scope [28-34]. In this paper, we used the CHNRI method [20-22]. We sought to identify a ranked set of COPD research priorities that need to be addressed in the next 10 years to substantially reduce the global impact of COPD - on patients, their families and society at large.

\section{METHODS}

\section{The CHNRI method}

This was a priority-setting study that crowdsourced expert opinion in a systematic way. The CHNRI method is a systematic, transparent, and democratic approach to priority setting for research and health interventions based on collective opinion. It employs the principle of crowdsourcing to collate and score research ideas against a pre-defined set of criteria. Based on submitted opinions of a larger number of experts, funders and policymakers are able to view the strengths, weaknesses, and relative ranking of each proposed research idea. While it allows researchers to independently generate and score research questions, it also involves relevant stakeholders, including patients, carers and support groups, at an early stage of the process, ensuring their ownership in the outcomes [35-41]. Previous experiences with more than a 100 conducted CHNRI exercises are summarized in Box 1.

\section{Management group}

We established a Management Group affiliated with National Institute of Health Research's RESPIRE Global Health Unit and Centre for Global Health at the Usher Institute, the University of Edinburgh, to identify research priorities to address the global burden of COPD. In September 2019, we developed a protocol to guide this process in line with recently published revised guidelines for the application of the CHNRI method, based on the experience of its use [35-41]. A small Management Group that includes the authors of this report (IR, DA, and AS) coordinated the steps of the priority setting exercise. 
The CHNRI method has been used in over 100 studies led by multilateral organisations (eg, World Health Organization (WHO), United Nations Children's Fund (UNICEF)), national governments (eg, China, India, and South Africa), and funders (eg, The Bill and Melinda Gates Foundation) to set research priorities in areas ranging from the reduction of global child mortality, chronic non-communicable diseases or disability to the efficient execution of national health plans [23,35-41]. Previous experiences and statistical simulations found considerable convergence of collective expert opinion leading to stable and replicable results [40].

To conduct this exercise, in September 2019 we ran searches on Web of Science's Core Collection to identify experts (ie. first, last or corresponding lead authors of the top 1\% most cited research articles) using the keywords "COPD" or "Chronic Obstructive Pulmonary Disease". Then, in January 2020, to put our findings in context of the wider literature, we ran additional searches on MEDLINE, Embase, Global Health, and CINAHL to identify studies on COPD priority settings published up to December 2019. We used a combination of Medical Subject Headings (MeSH) on the databases as follows: "* pulmonary disease, chronic obstructive/ or *bronchitis, chronic/ or * pulmonary emphysema/" AND "*morbidity/ or *mortality/ *risk factors/" AND burden adj3 disease" AND "Health Priorit*/ or priority setting*/or Resource allocation/”. No language or geographical restrictions were applied.

Although we took steps to maximise response rate using a three-stage invitation process (initial contact, invitation to submit ideas, and invitation to submit scores), and a follow-up after four weeks for non-response, the overall response rate can be considered quite low, and it falls in line invitation to participate in a research without incentives, also observed in previous CHNRI exercises [23-25].

\section{Invitation of experts}

We identified 432 experts in COPD research from across the world by searching the Web of Science's Core Collection for the most productive authors in the preceding five-year period, or those who were lead authors (first, last or corresponding) of the top 1\% most cited research articles. Search strategies and selection criteria are summarized in Box 1. In the first phase of invitation, e-mails were sent to all the experts seeking their participation and with details of the objectives and context of the exercise. In a second e-mail, experts that showed interest were invited to generate a minimum of three research questions. The Management Group then scrutinised the submitted ideas and ensured that the wording of each idea fitted the format for the scoring process. This led to a consolidated list of 230 unique research ideas, that could be categorised in 6 sub-themes (Box 2). Then, the experts were re-invited (with a four-week timeline for follow-up) to systematically rank these ideas using pre-agreed criteria, which are listed below.

\section{Box 2. Research sub-themes}

\section{Six broad research sub-themes were identified:}

I. Epidemiology and risk factors of COPD (26 research ideas).

II. Aetiology and pathophysiology of COPD and exacerbations (43 research ideas).

III. Strategies (and policies) for prevention, management, and rehabilitation of COPD (92 research ideas).

IV. COPD self-management and adherence to treatment (10 research ideas).

V. Approaches towards improved diagnosis and classification of COPD (44 research ideas).

VI. Monitoring disease progression and/or prognosis of COPD (15 research ideas).

\section{Research context and criteria}

The context and the criteria for scoring were defined in line with recommendations from the previous exercises and guidelines $[38,41]$. The context was defined as "global", ie, taking into account that the majority of the burden is in LMICs. The timeframe within which the results were expected from proposed research was specified as up to 10 years. The age group of people with COPD was defined as 40 years or older, because the management group agreed that the overwhelming majority of COPD cases would be affecting this age group. The target population include respiratory physicians, researchers, policymakers, funders, patients and support groups across various global settings.

Six independent criteria were agreed by the Management Group and used to discriminate between the many proposed research questions identified:

1. Answerability: Is this research question likely to be answered using the proposed methods and approaches?

2. Effectiveness: Is this research question likely to lead to interventions that will effectively reduce the burden of COPD over time? 
3. Feasibility: Is it feasible to address this research question given the existing level of knowledge, capacity and resources?

4. Deliverability: Is this research question likely to lead to interventions or solutions that could be readily implemented and deliverable to population at scale?

5. Burden reduction: Is this research question likely to lead to a significant reduction in COPD burden?

6. Equity: Is this research question likely to reduce inequity in the population?

Scoring and analysis

All invited contributors were asked to score each submitted research question using these pre-defined criteria. Experts were offered four response options for scoring: 0 (unlikely to meet the criterion); 0.5 (not sure if it can meet the criterion); 1 (likely to meet the criterion); or left blank if the expert felt insufficiently informed to make a judgment. We generated intermediate scores by calculating the mean of the individual scores for each research question and each criterion received from all experts, and it ranged from 0\%-100\%. Subsequently, the overall Research Priority Score (RPS) assigned to each research question was a simple mean of all six criteria-specific scores. Average Expert Agreement (AEA), an indicator of the average proportion of scorers that returned the most common answer for a research question was also calculated for each research question to provide an understanding of the level of agreement among scorers. This is expressed as the frequency of the mode (ie, the most common score divided by the total number of scores).

$$
A E A=\frac{1}{5} \times \sum_{q=1}^{5} \frac{N(\text { Scorers that provided most frequent response })}{N(\text { Scorers })}
$$

where " 5 " represents the five criteria, and " $N$ " is the total number of experts.

\section{RESULTS}

Among the 432 researchers contacted, 64 (14.8\%) contributed 264 research questions. We removed the duplicates and consolidated the final list into 230 research questions, which were scored by 34 researchers according to the six criteria of interest. A total of 8 experts from LMICs accepted our invitation and took part in this exercise, although of the initial 432 experts contacted, over 30\% were indirectly affiliated or collaborating with researchers in LMICs. Further information on the 64 contributors of research questions and 34 among them who also agreed to take the time and score is provided in the Online Supplementary Document, in accordance to General Data Protection Regulation.

\section{Research priority scores and expert agreement}

The overall RPS for the 230 research questions ranged from 0.868 (highest) to 0.243 (lowest). The AEA revealed that across the entire exercise, on average, $43 \%-82 \%$ of scorers provided the same most common answer to a criterion related to the 230 proposed research ideas (Supplementary Online Material). The AEA decreased with decreasing RPS (Spearman's rho $(\rho)=0.7907, P<0.001$ ) suggesting a higher degree of agreement among experts on the top ranked questions and consistent with previous CHNRI findings. See Tables S1-S7 in the Online Supplementary Document for RPS and AEA for all 230 research ideas.

\section{Top-ranked priorities}

The top-ranked research priority proposed to develop new effective strategies for smoking cessation. Among the top 20 overall research priorities, six were focused on feasible and cost-effective pulmonary rehabilitation delivery and access, particularly in primary care and low-resource settings. Three of the top 10 overall priorities called for research on improved screening and better diagnostic methods for COPD in primary care and low-resource settings. Further ideas that drew support from scorers involved a better understanding of risk factors for COPD, development of effective training programmes and guidelines for health workers and physicians in low resource settings, and encouraging physical activity (Table 1).

\section{Bottom-ranked priorities}

Because of very poor scores on one or more of the six criteria, the scorers showed the lowest collective optimism towards ideas that proposed to identify biological pathways that underlie different clinical presentations, then exploring if COPD with airway mucus hypersecretion and higher risk of death should be approached 
as a separate disease and the basis of day-to-day variability in symptoms. Also, there was low optimism towards highly specific questions that sought to provide answers of limited use and transferability or contribute to refining the existing definitions of COPD and exacerbation. Interestingly, there was not much support for studying gene reprogramming of the epithelium in COPD, piloting replacement of a "COPD" diagnosis with individual clinical and biological phenotyping, evaluating the usefulness of measuring sensory and affective dimension of acute or episodic breathlessness, or evaluating animal models to determine the contributions of a new generation of nicotine products. Finally, the least enthusiasm was shown towards synthesising various lines of evidence to reach a consensus whether COPD is a disease or a disorder (Table 2).

\section{Top-ranked priorities across research criteria}

When research ideas were considered by their likelihood of answerability, "Studying whether inhaled corticosteroids increase risk of bacterial infections in COPD" received the maximum score. For likelihood of effectiveness, there was very high agreement that developing new strategies (including new combinations of pharmacological and non-pharmacological strategies) to improve smoking cessation would be the most effective research idea. Based on the likelihood of feasibility, the leading research idea was improved understanding of COPD risk factors and their association with COPD incidence and exact effects, studying whether inhaled corticosteroids increase the risk of bacterial infections in COPD, and identifying optimal screening methods for COPD in primary care. When likelihood of deliverability was analysed, identifying optimal diagnostic approaches for COPD in low-resource settings was ranked as most deliverable, followed by identifying optimal screening methods for COPD in primary care. The greatest impact on COPD burden was associated with the idea of identifying strategies that are effective and cost-effective in reducing anxiety and depression among individuals with COPD. Finally, most of research ideas in the top 10 when considered by their likelihood of improving equity in the population were focused on low resource settings (Table 3). Please refer to the Online Supplementary Document for further details of top-ranked priorities and scores across each of the criteria.

\section{DISCUSSION}

\section{Main findings}

On the basis of the six suggested criteria, the top priority was focused on finding acceptable, effective and cost-effective ways for smoking cessation, which is research in risk reduction. Furthermore, three of the top 10 overall priorities called for research on improved screening and accurate diagnostic methods for COPD in primary care and low resource settings [42]. Moreover, six of the 20 top priorities were focused on feasible and cost-effective pulmonary rehabilitation delivery and access, particularly in primary care and low resource settings, which highlights the relevance of this exercise for LMICs. Other ideas that drew support from participants involved better understanding of risk factors for COPD, development of effective training programmes and guidelines for health workers and physicians in low resource settings and encouraging physical activity. The main output of the CHNRI process is an intuitive list of meaningful research questions provided by a group of 34 experts, with 230 research ideas organised and ranked according to explicit priority criteria. The COPD experts who took part in this CHNRI exercise concurred that most of these key research questions may be successfully answered by 2030 .

\section{Findings in the context of the literature}

A key difference between this process and those used in other prioritisation exercises, eg, the American Thoracic Society/European Respiratory Society statement on research questions in COPD [32] is that, in previous attempts to define research priorities, they were not systematically compared. Recommendations for research were usually listed as a result of broad overall consensus of participants following the discussion. Furthermore, the International Primary Care Respiratory Group (IPCRG) conducted an e-Delphi exercise, where diagnosing COPD in a primary care setting was a priority after that survey was completed. A notable difference was that community-based pulmonary rehabilitation was lower down the list and there was a separate category for tobacco dependence [33].

Meanwhile, in a UK priority setting for respiratory research involving physicians, researchers and professional societies [43], the leading focus leaned towards basic science including lung development and ageing, lung injury, repair and regeneration, susceptibility to infections, and sleep apnoea syndromes, which are not in our top priorities for reasons already discussed above. In an exercise involving only patients with COPD and asthma, priorities highlighted focused primarily on aetiology, co-morbidity and effective medication [44]. Anoth- 


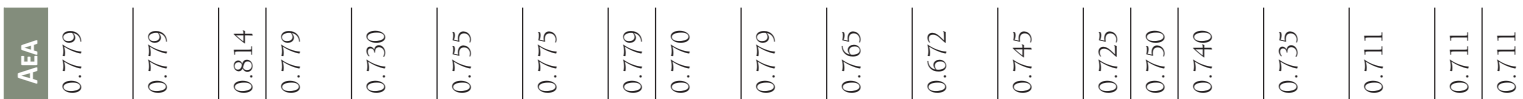

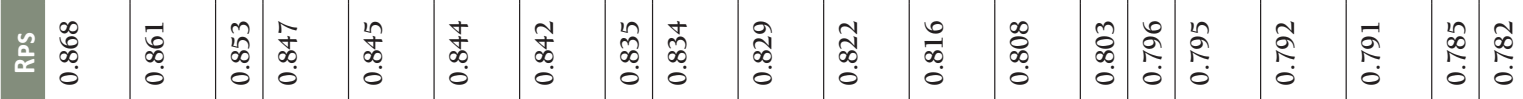

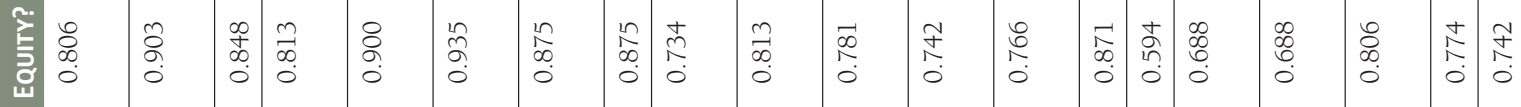

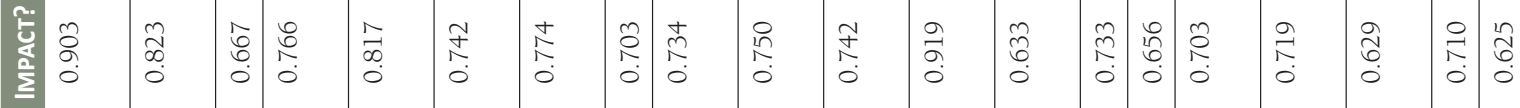
咅

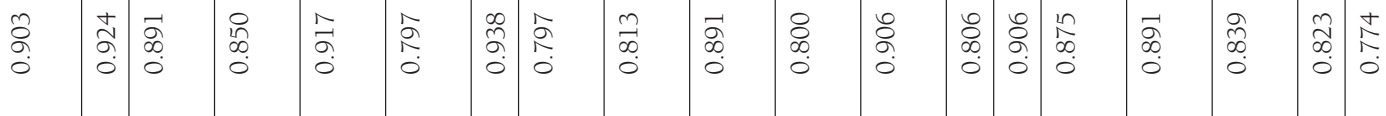

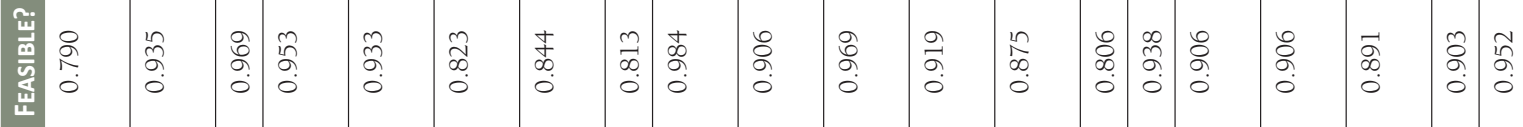

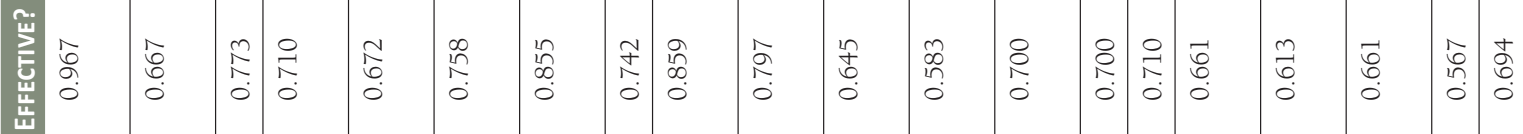

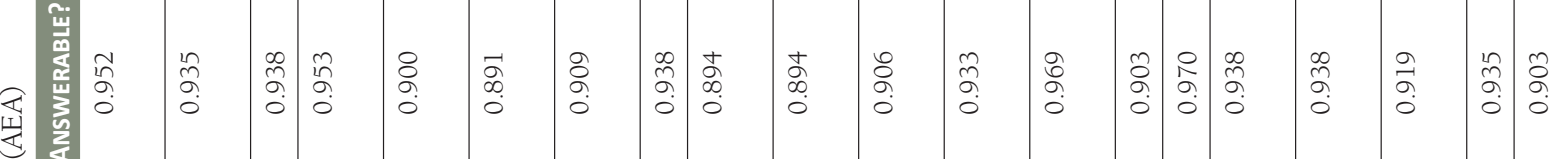
密

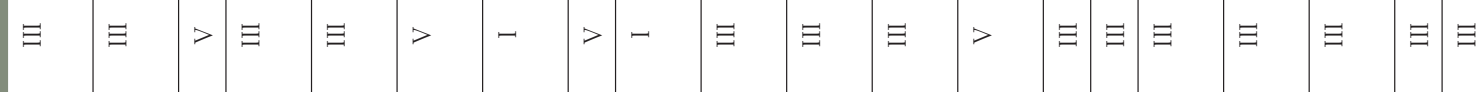
商 苛 安 $\bar{\varpi}$ 的

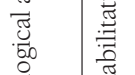

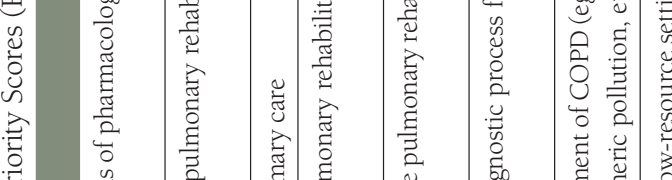

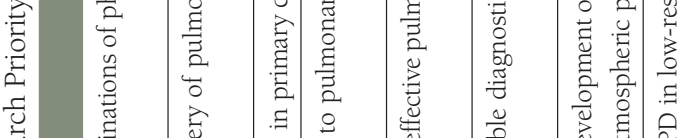

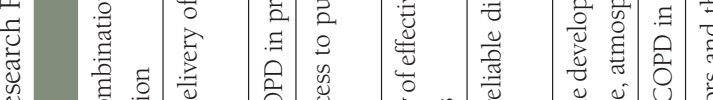

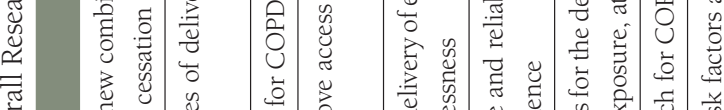

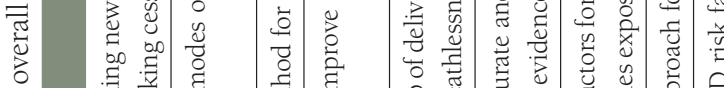

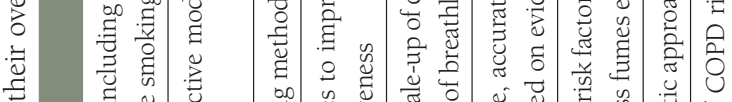

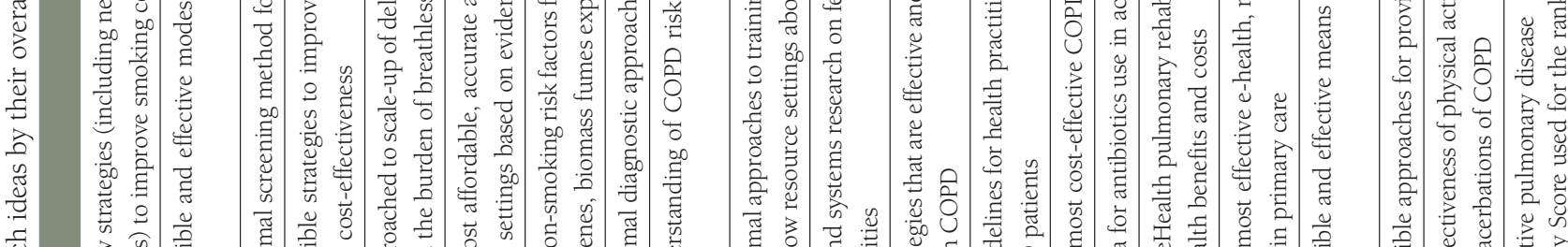

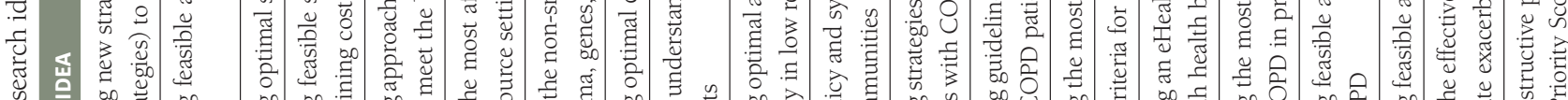

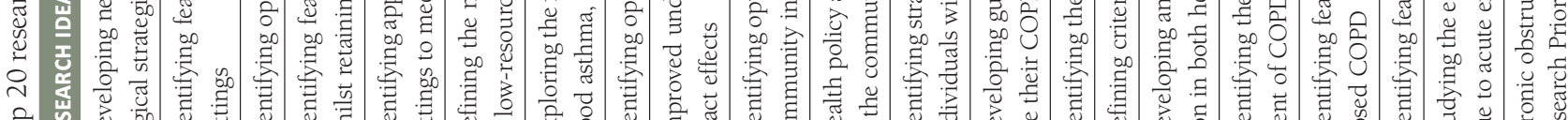

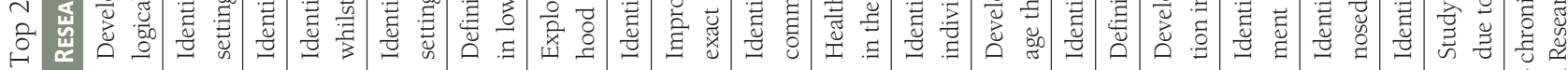

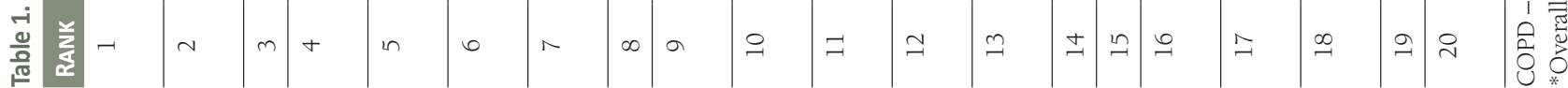




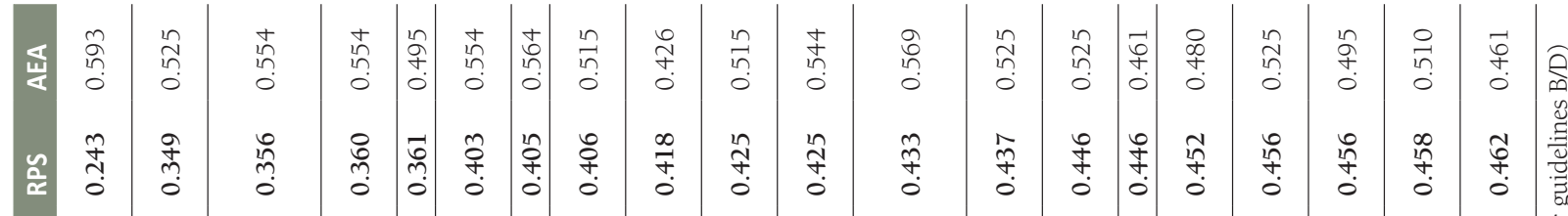

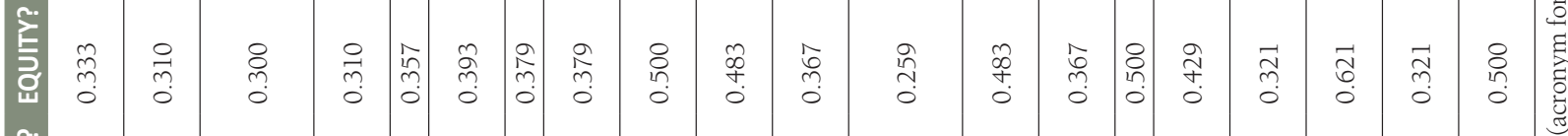

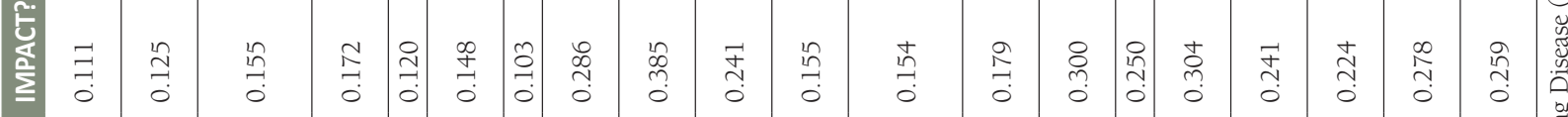

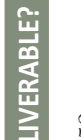

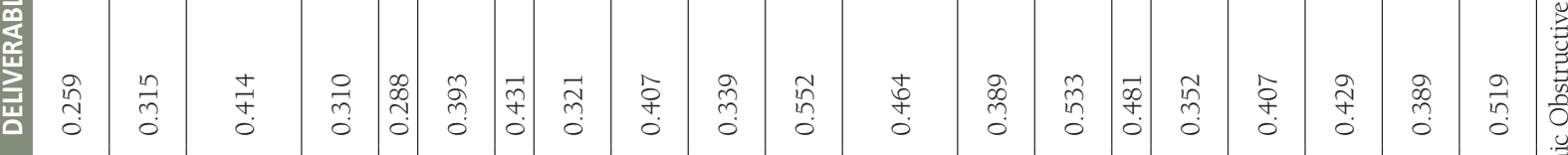

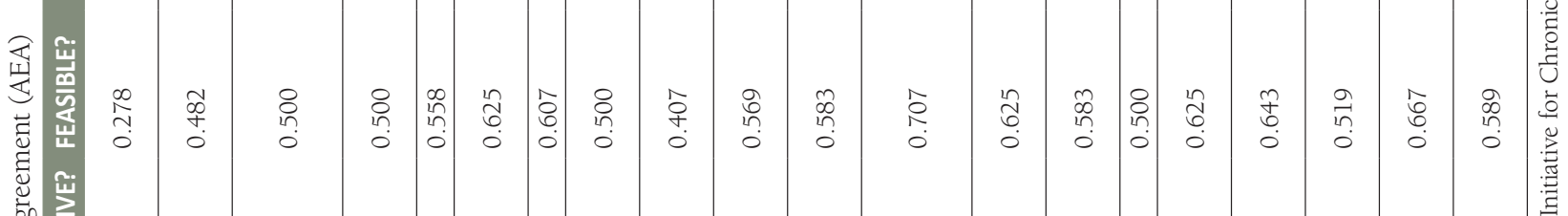

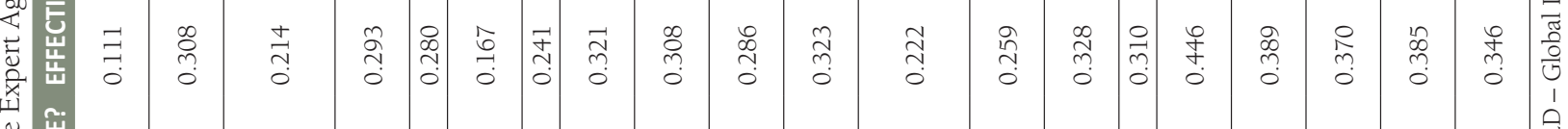

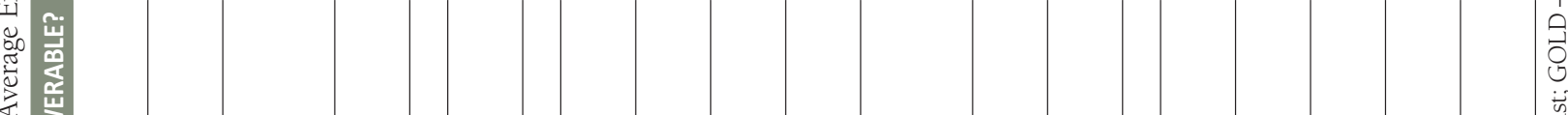

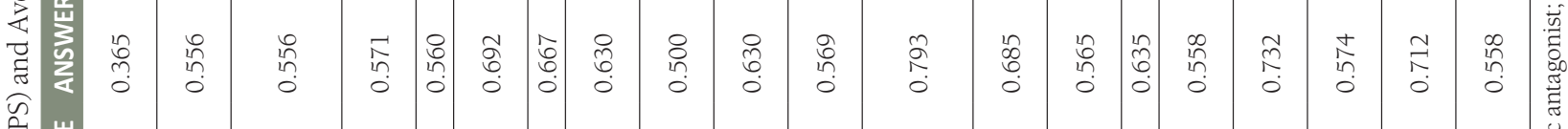

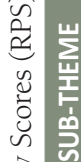

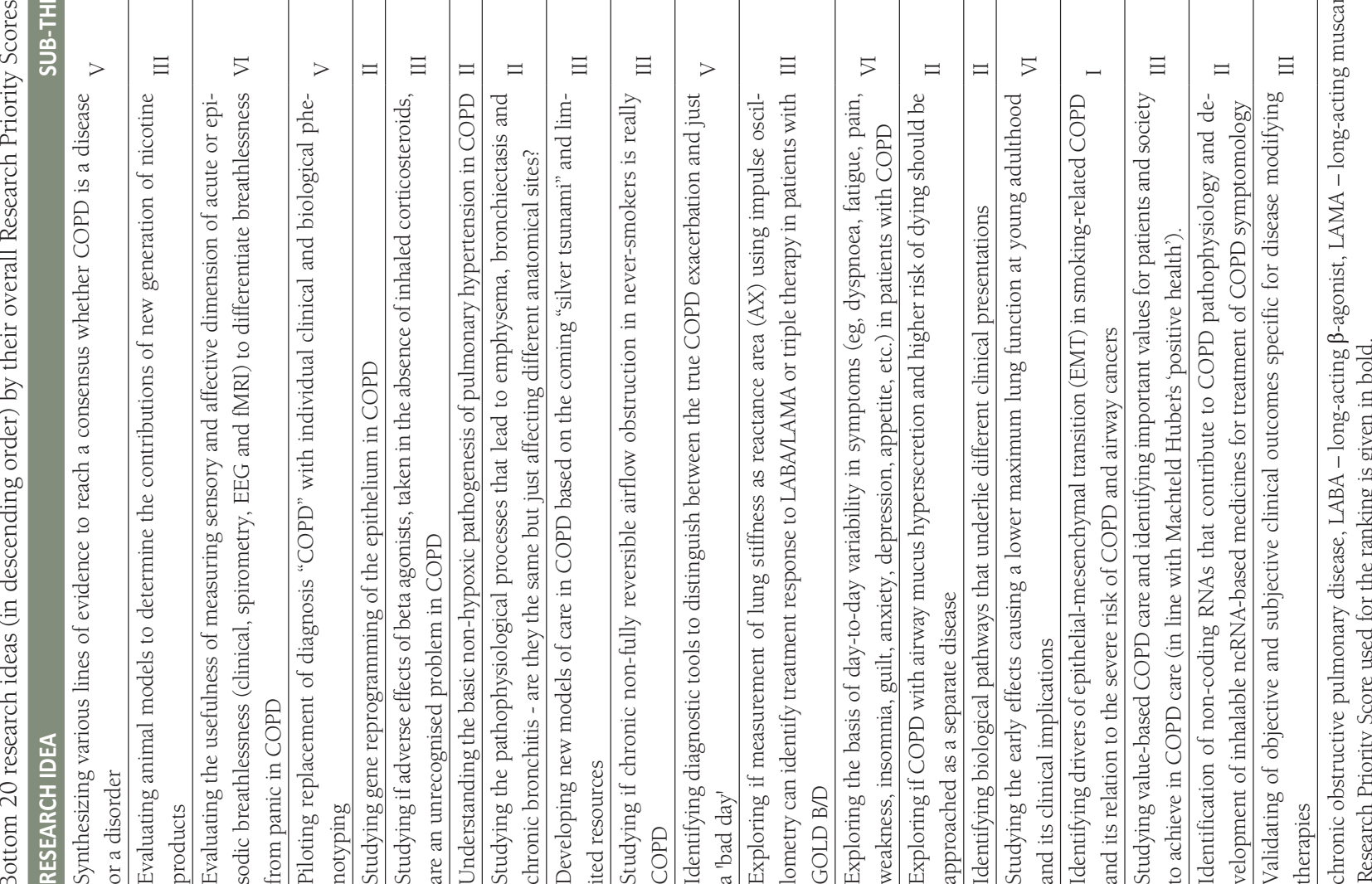

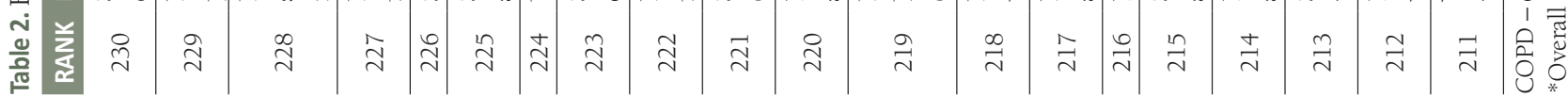




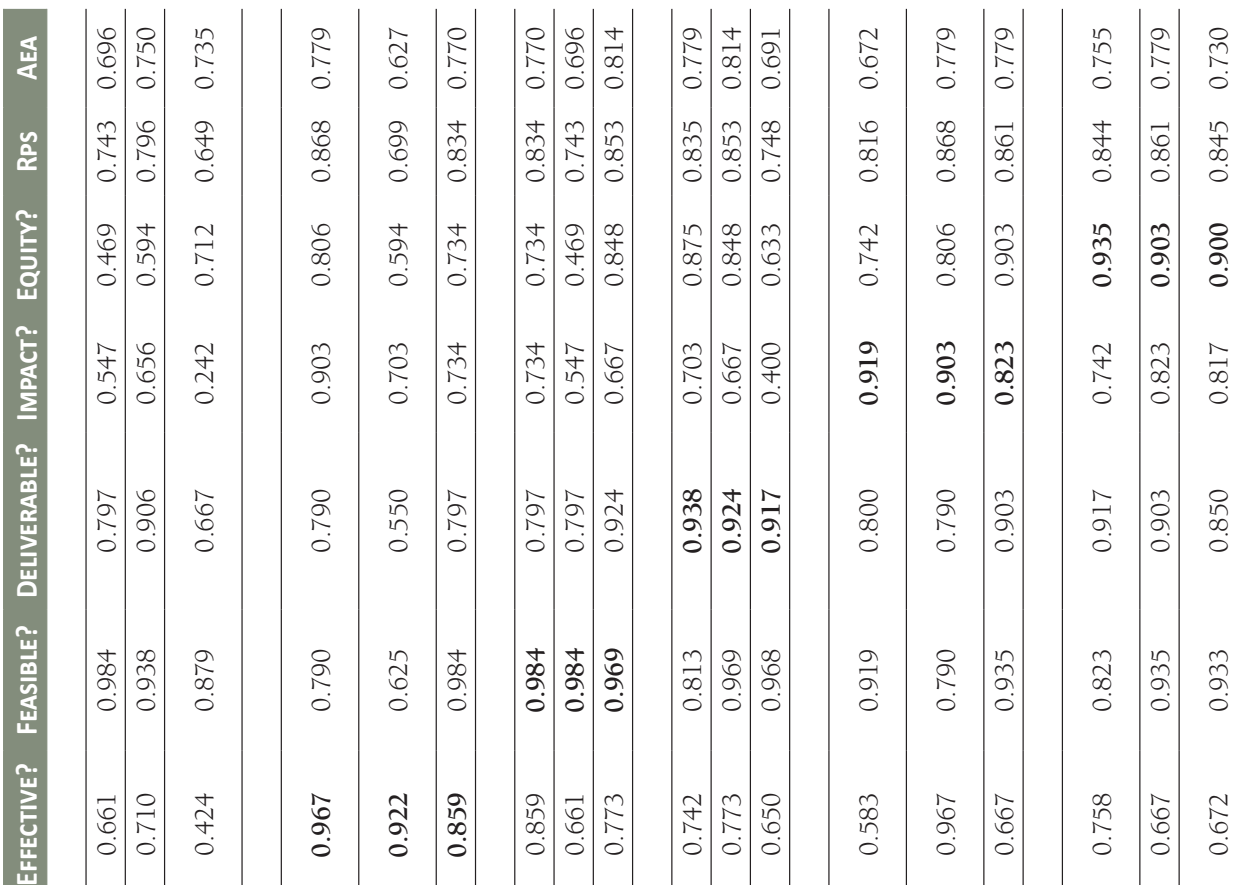

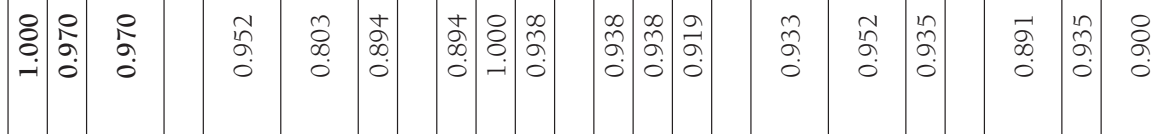
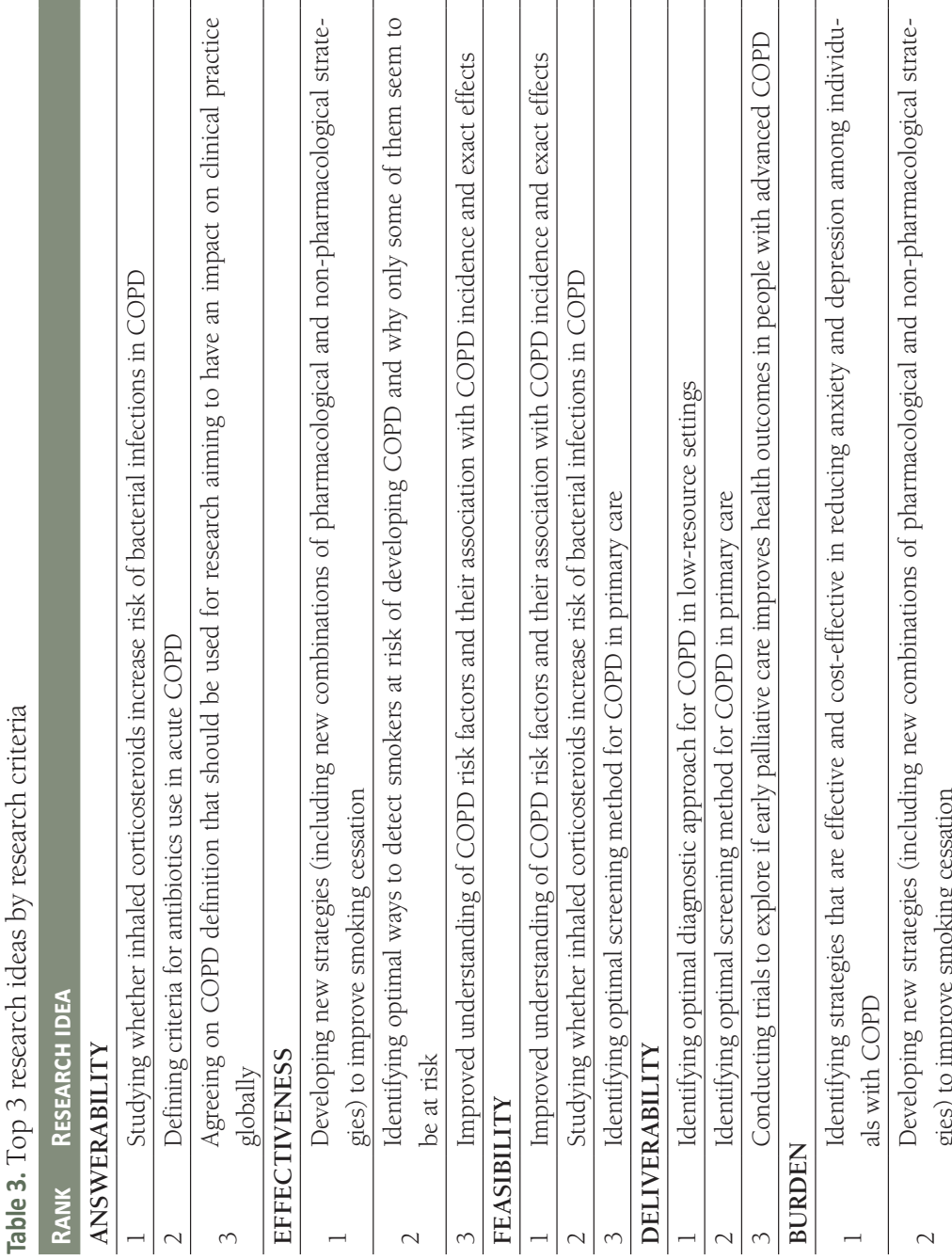

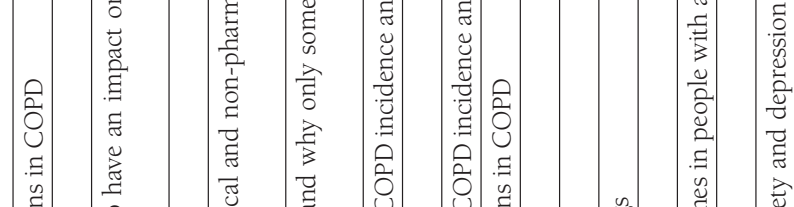

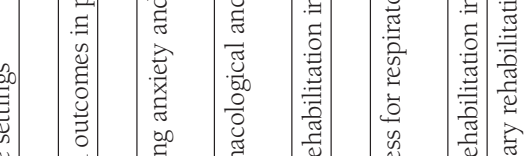

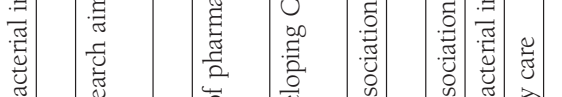

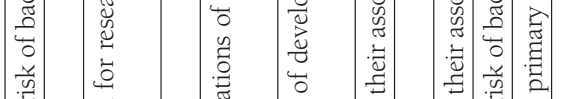

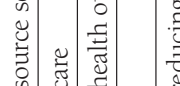

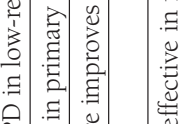

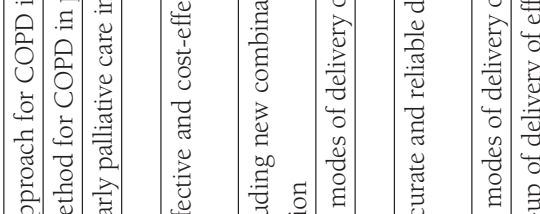

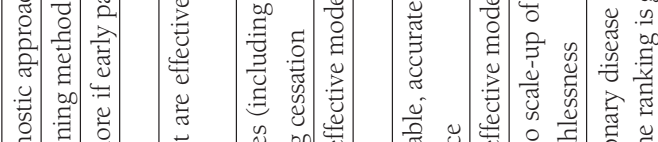

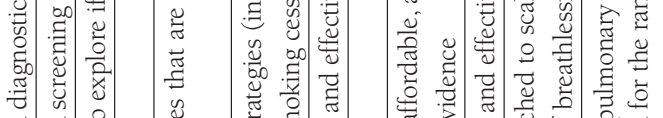

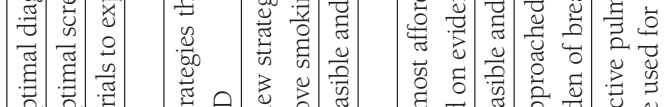

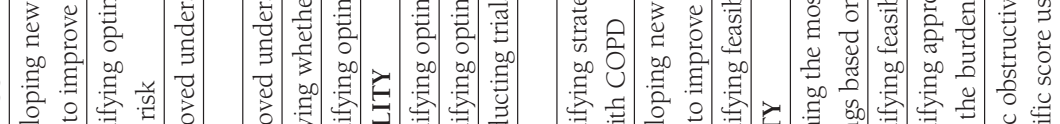

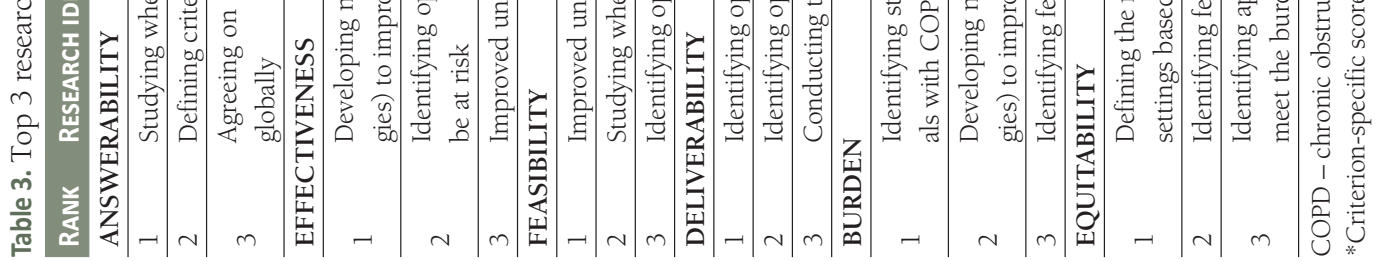


er priority study among patients mainly emphasized the need to improve the ability to exercise among adults living with COPD [45]. Although both patients' studies [44,45] employed no systematic measure of collating, analysing and ranking ideas, their findings reflect in our overall top priorities, and specifically for priorities listed under deliverability which largely focuses on pulmonary rehabilitation and physical activity, and addressing screening, diagnosis and treatment in primary care settings to improve outcomes, respectively.

\section{Research and policy implications}

The experts agreed that the most pressing feasible research questions to address in the next decade for COPD reduction were on prevention, diagnosis and rehabilitation of COPD, especially in low resource settings. This appears to be in line with a number of recommendations from respiratory experts, giving credence to this exercise. For example, in a recent experts' statement on the top ten research questions for improving COPD care in the next decade [46], they proposed exploring impact of new forms of electronic cigarettes on COPD burden, addressing challenges with COPD diagnosis, patient's classification and risk stratification, and improvements in non-pharmacologic management particularly pulmonary rehabilitation, as top priorities, which clearly reflect in the our findings and support our recommendations for COPD research in the next decade. Important overall messages from the top of the list imply that LMIC settings should increasingly become a priority for research on COPD, because large majority of COPD-related deaths in the world today occur there. This implies an increased focus on low-cost preventive diagnostic and therapeutic measures, including tobacco reduction and increase in physical exercise.

Therefore, research on proper, cost-effective implementation of those measures will become increasingly important, which will require extensive capacity building in low-resource settings. Such research should be expected to lead to tailored country guidelines on addressing COPD, which are much needed [47]. Finally, the role of stress is still rather uncertain, but certainly worth investigating in future studies (Box 3) [48-50].

Box 3. Possible role of stress

Box topic not identified by the CHNRI method, which could be important to investigate for several reasons, is stress. Stress is a wellknown trigger of inflammatory activity $[48,49]$, and affects clinical outcomes in COPD [50], meaning that it is clinically relevant in this context, thus making stress a possible treatment target that could have benefits for COPD and beyond. Meanwhile, atmospheric pollution was broadly ranked as a non-smoking priority in the overall top 10 research priorities and also listed in the top ten by likelihood of effectively reducing the burden of COPD over time. However, it may be somewhat surprising that other specific areas of research on air quality particularly in LMICs, were not given more priority. After cigarette smoking, using biomass for cooking and the resulting indoor pollution is a major cause of COPD in less developed countries, where both indoor and outdoor air pollution are a concern worth studying further. Also, work on the development of pharmacological treatment or treating exacerbations did not feature prominently. Finally, although there were no specific questions about COVID-19 because the protocol for this exercise was developed in September 2019, the findings would still be relevant, and helpful at a follow-up to this exercise, in view of a likely risk for poor health outcomes among COVID-19 patients with COPD and other chronic airways diseases.

\section{Strengths and limitations}

Diverse experts from across the world have come together for the first time to identify research priorities, which now need to be built on through strategic research investment in these prioritised areas. Nevertheless, some limitations are worth noting. The application of the CHNRI methodology to the field of COPD required some contextual adaptations including the experts and the scorers' selection, question consolidation, modified criteria and scoring processes. Still, it is important to note that the CHNRI methodology was originally conceived to be adaptive and has been similarly customised before and used in various settings [23], and our adaptations were collectively discussed and agreed upon by the Management Group. Another deviation from the prescribed process was lack of involvement from the representatives of the funders from the early stages.

In terms of equity, diversity and inclusivity, we carefully studied if the process inherently biased the outcomes against some groups or types of research questions. Table 2 presented research questions that achieved the lowest scores. It is clear that highly theoretical work was not favoured in light of the urgent need to address the COPD burden globally, especially in LMICs. Besides, the criteria were chosen to prefer research questions that are feasible and could realistically address the burden within a decade. This did not favour research questions from basic science. This is why the questions at the bottom of the list were often highly specific, or advanced research ideas with more "blue-sky" thinking, or they had complex downstream outcomes.

One possible reason that could have disadvantaged highly specific or innovative research questions could be that some experts were not necessarily familiar enough with the research area to recognise a potentially feasible research question that is suitable for further exploration. It is also possible that professional expertise and 
clinical background of the experts affected the results. Furthermore, only COPD researchers were included, while other physicians (including those in primary care), nurses, patients, support groups and funding bodies, who are all important stakeholders, were not.

Despite efforts to represent all geographic regions and resource levels, respondents were predominantly COPD researchers from developed countries. We note that the South East Asia, African and Eastern Mediterranean regions were relatively under-represented. Although this likely reflects the current low levels of COPD research and advocacy efforts and potential barriers to participation that may exist in these settings, we acknowledge that priorities may vary by culture, region and resource level. This exercise was not able to capture and examine these differences, and culture and system specific research may be required.

Another important contributing factor for under-representation of LMIC-based researchers is likely the inclusion criterion of very high productivity or citations of their papers, which is difficult for them to achieve given limited resources for research, although our initial searches would have returned a sizeable number of researchers in HICs who were collaborating with colleagues in LMICs. This indeed reflects in our final top 20 rankings, with six focused on feasible and cost-effective pulmonary rehabilitation delivery and access in low resource settings, and three of the top 10 calling for research on improved screening and accurate diagnostic methods for COPD in primary care and low resource settings, both clearly important needs in LMICs. Moreover, seven of the research ideas in the top 10 under the likelihood of improving equity in the population were directly focused on LMICs (see Table S6 in the Online Supplementary Document). The drop-out rate from providing research questions to scoring them was also expected to be quite high. This is common with prioritisation exercises based on crowdsourcing, and it should be acknowledged, which was elaborated in Box 1 [23-25].

\section{CONCLUSIONS}

Research priorities identified by this systematic international process should inform and motivate policymakers, funders and researchers to support and conduct research to reduce the global burden of COPD. The largest gains should be expected in LMIC settings, as the large majority of COPD deaths occur in those settings [51]. The follow-up to this process should identify and map outcomes to currently funded research to highlight apparent gaps and opportunities for increased investment.

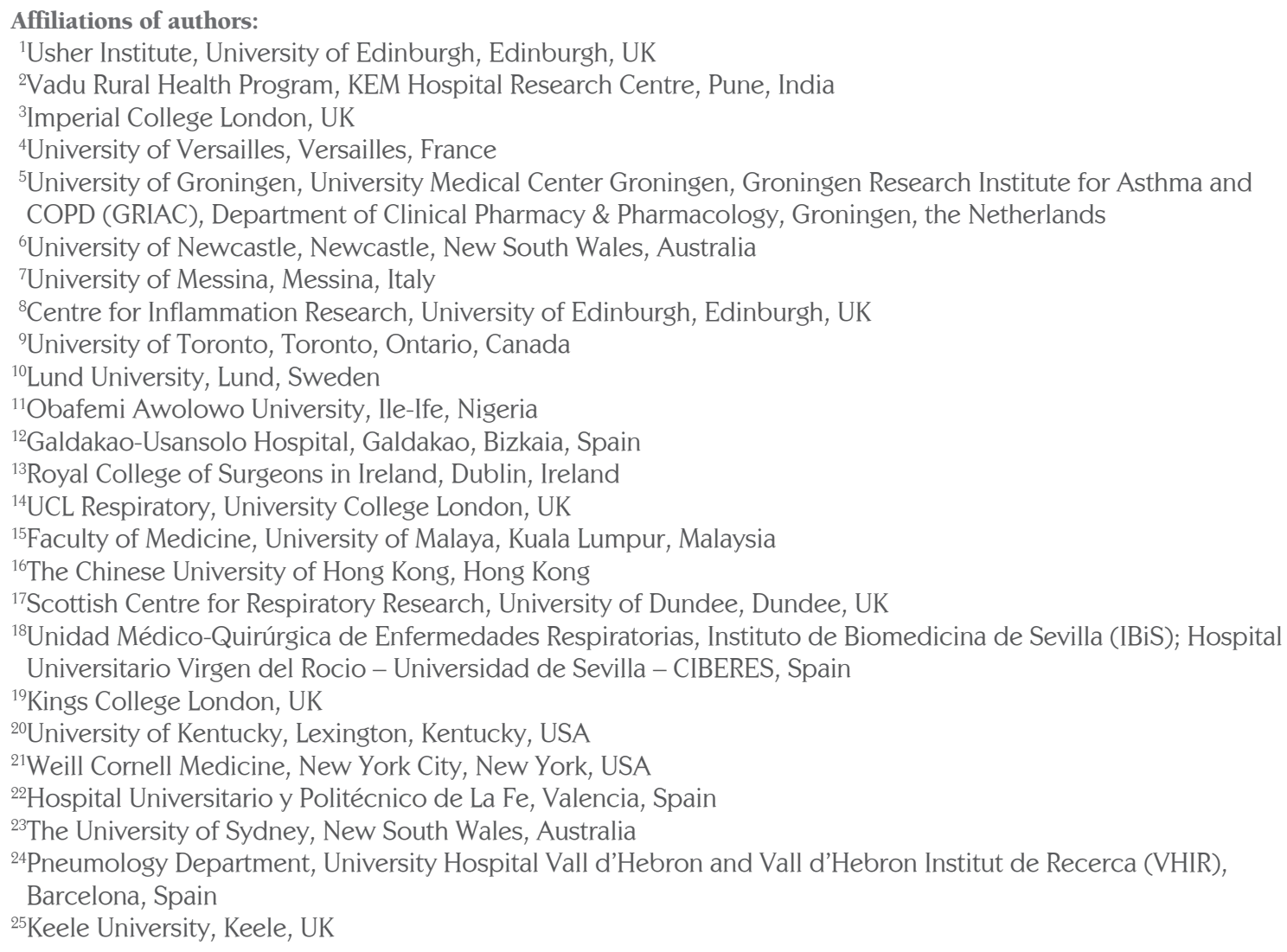


${ }^{26}$ Bone-metabolic Research Unit, Copenhagen University Hospital Rigshospitalet, Denmark

${ }^{27}$ Cousins Center for Psychoneuroimmunology and Department of Psychiatry and Biobehavioral Sciences,

University of California, Los Angeles, California, USA

${ }^{28}$ School of Public Health, Zhejiang University School of Medicine, Hangzhou, China

${ }^{29}$ Women's and Children's Hospital, Adelaide, South Australia, Australia

${ }^{30}$ Pulmonary Research Institute at Lungen Clinic Grosshansdorf, Airway Research Center North (ARCN), German

Centre for Lung Research (DZL), Germany

${ }^{31}$ Centre for Cardiovascular Science, The Queen's Medical Research Institute, University of Edinburgh,

Edinburgh, UK

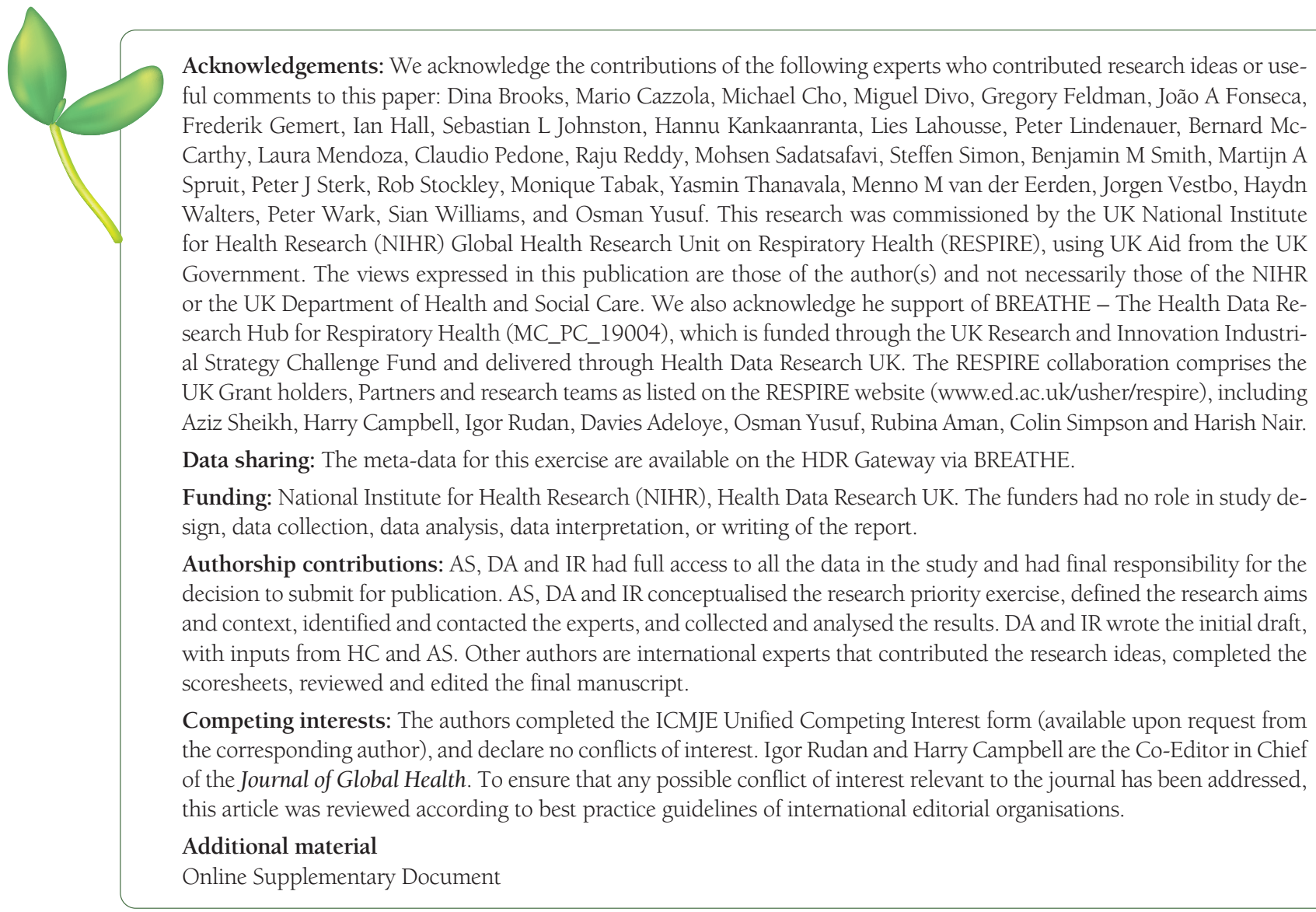

1 Adeloye D, Chua S, Lee C, Basquill C, Papana A, Theodoratou E, et al; Global Health Epidemiology Reference Group (GHERG). Global and regional estimates of COPD prevalence: Systematic review and meta-analysis. J Glob Health. 2015;5:020415. Medline:26755942 doi:10.7189/jogh.05.020415

2 Vos T, Flaxman AD, Naghavi M, Lozano R, Michaud C, Ezzati M, et al. Years lived with disability (YLDs) for 1160 sequelae of 289 diseases and injuries 1990-2010: a systematic analysis for the Global Burden of Disease Study 2010. Lancet. 2012;380:2163-96. Medline:23245607 doi:10.1016/S0140-6736(12)61729-2

3 Chronic obstructive pulmonary disease (COPD) Fact sheet N 315". Geneva: World Health Organization; 2015. Archived from the original on 2016-03-04.

4 Brakema EA, Tabyshova A, van der Kleij RMJJ, Sooronbaev T, Lionis C, Anastasaki M, et al. The socioeconomic burden of chronic lung disease in low-resource settings across the globe - an observational FRESH AIR study. Respir Res. 2019;20:291. Medline:31864411 doi:10.1186/s12931-019-1255-z

5 Beran D, Zar HJ, Perrin C, Menezes AM, Burney P. Forum of international respiratory societies working group C: Burden of asthma and chronic obstructive pulmonary disease and access to essential medicines in low income and middle-income countries. Lancet Respir Med. 2015;3:159-70. Medline:25680912 doi:10.1016/S2213-2600(15)00004-1

6 Leung JM, Niikura M, Yang CWT, Sin DD. COVID-19 and COPD. Eur Respir J. 2020;56:2002108. Medline:32817205 doi:10.1183/13993003.02108-2020

7 Vogelmeier CF, Criner GJ, Martinez FJ, Anzueto A, Barnes PJ, Bourbeau J, et al. Global Strategy for the Diagnosis, Management and Prevention of Chronic Obstructive Lung Disease 2017 Report: GOLD Executive Summary. Respirology. 2017;22:575601. Medline:28150362 doi:10.1111/resp.13012 
8 Lozano R, Naghavi M, Foreman K, Lim S, Shibuya K, Aboyans V, et al. Global and regional mortality from 235 causes of death for 20 age groups in 1990 and 2010: a systematic analysis for the Global Burden of Disease Study 2010. Lancet. 2012;380:2095-128. Medline:23245604 doi:10.1016/S0140-6736(12)61728-0

9 Williams S, Sheikh A, Campbell H, Fitch N, Griffiths C, Heyderman RS, et al; Global Health Respiratory Network. Respiratory research funding is inadequate, inequitable, and a missed opportunity. Lancet Respir Med. 2020;8:e67-8. Medline:32763207 doi:10.1016/S2213-2600(20)30329-5

10 World Health Organization. The 10 leading causes of death in the world, 2000 and 2011. Archived from the original on December 2, 2013. Available: https://www.who.int/news-room/fact-sheets/detail/the-top-10-causes-of-death. Accessed: 29 November 2020.

11 Rycroft CE, Heyes A, Lanza L, Becker K. Epidemiology of chronic obstructive pulmonary disease: a literature review. Int J Chron Obstruct Pulmon Dis. 2012;7:457-94. Medline:22927753 doi:10.2147/COPD.S32330

12 GBD Chronic Respiratory Disease Collaborators. Prevalence and Attributable Health Burden of Chronic Respiratory Diseases from 1990-2017: A systematic analysis from the Global Burden of Disease Study 2017. Lancet Respir Med. 2020;8:585-96. Medline:32526187 doi:10.1016/S2213-2600(20)30105-3

13 Lomborg B. Global problems, local solutions: costs and benefits. Cambridge: Cambridge University Press; 2013.

14 Bloom DE, Cafiero E, Jané-Llopis E, Abrahams-Gessel S, Bloom LR, Fathima S, et al. The global economic burden of noncommunicable diseases. Geneva: World Economic Forum; 2011.

15 Decramer M, Janssens W, Miravitlles M. Chronic obstructive pulmonary disease. Lancet. 2012;379:1341-51. Medline:22314182 doi:10.1016/S0140-6736(11)60968-9

16 Rabe KF, Watz H. Chronic obstructive pulmonary disease. Lancet. 2017;389:1931-40. Medline:28513453 doi:10.1016/ S0140-6736(17)31222-9

17 Varmus H, Klausner R, Zerhouni E, Acharya T, Daar AS, Singer PA. Public health. Grand Challenges in Global Health. Science. 2003;302:398-9. Medline:14563993 doi:10.1126/science.1091769

18 Daar AS, Singer PA, Persad DL, Pramming SK, Matthews DR, Beaglehole R, et al. Grand challenges in chronic non-communicable diseases. Nature. 2007:450:494-6. Medline:18033288 doi:10.1038/450494a

19 Collins PY, Patel V, Joestl SS, March D, Insel TR, Daar AS, et al. Grand challenges in global mental health. Nature. 2011;475:2730. Medline:21734685 doi:10.1038/475027a

20 Rudan I, El Arifeen S, Black RE, Campbell H. Childhood pneumonia and diarrhoea: setting our priorities right. Lancet Infect Dis. 2007;7:56-61. Medline:17182344 doi:10.1016/S1473-3099(06)70687-9

21 Rudan I. Global health research priorities: mobilizing the developing world. Public Health. 2012;126:237-40. Medline:22325672 doi:10.1016/j.puhe.2011.12.001

22 Yoshida S, Martines J, Lawn JE, Wall S, Souza JP, Rudan I, et al. Setting research priorities to improve global newborn health and prevent stillbirths by 2025. J Glob Health. 2016;6:010508. Medline:26401272

23 Rudan I, Yoshida S, Chan KY, Sridhar D, Wazny K, Nair H, et al. Setting health research priorities using the CHNRI method: VII. A review of the first 50 applications of the CHNRI method. J Glob Health. 2017;7:011004. Medline:28685049 doi:10.7189/jogh.07.011004

24 Tomlinson M, Swartz L, Officer A, Chan KY, Rudan I, Saxena S. Research priorities for health of people with disabilities: an expert opinion exercise. Lancet. 2009;374:1857-62. Medline:19944866 doi:10.1016/S0140-6736(09)61910-3

25 Shah H, Albanese E, Duggan C, Rudan I, Langa KM, Carrillo MC, et al. Research priorities to reduce the global burden of dementia by 2025. Lancet Neurol. 2016;15:1285-94. Medline:27751558 doi:10.1016/S1474-4422(16)30235-6

26 Tomlinson M, Rudan I, Saxena S, Swartz L, Tsai AC, Patel V. Setting priorities for global mental health research. Bull World Health Organ. 2009;87:438-46. Medline:19565122 doi:10.2471/BLT.08.054353

27 Sheikh A, Rudan I, Cresswell K, Dhingra-Kumar N, Tan ML, Häkkinen ML, et al. World Health Organization's Management Team on Research Priorities for Medication Safety. Agreeing on global research priorities for medication safety: an international prioritisation exercise. J Glob Health. 2019;9:010422. Medline:30842883 doi:10.7189/jogh.09.010422

28 Krishnan JA, Lindenauer PK, Au DH, Carson SS, Lee TA, McBurnie MA, et al. COPD Outcomes-based Network for Clinical Effectiveness and Research Translation. Stakeholder priorities for comparative effectiveness research in chronic obstructive pulmonary disease: a workshop report. Am J Respir Crit Care Med. 2013;187:320-6. Medline:23155144 doi:10.1164/ rccm.201206-0994WS

29 Stellefson M, Alber J, Paige S, Castro D, Singh B. Evaluating Comparative Effectiveness Research Priorities for Care Coordination in Chronic Obstructive Pulmonary Disease: A Community-Based eDelphi Study. JMIR Res Protoc. 2015;4:e103. Medline:26268741 doi:10.2196/resprot.4591

30 Caron-Flinterman JF, Broerse JE, Teerling J, Bunders JF. Patients' priorities concerning health research: the case of asthma and COPD research in the Netherlands. Health Expect. 2005;8:253-63. Medline:16098155 doi:10.1111/j.1369-7625.2005.00337.x

31 Puhan MA, Yu T, Boyd CM, Ter Riet G. Quantitative benefit-harm assessment for setting research priorities: the example of roflumilast for patients with COPD. BMC Med. 2015;13:157. Medline:26137986 doi:10.1186/s12916-015-0398-0

32 Celli BR, Decramer M, Wedzicha JA, Wilson KC, Agustí AA, Criner GJ, et al. The ATS/ERS Task Force for COPD Research. An official American Thoracic Society/ European Respiratory Society statement: research questions in COPD. Eur Respir Rev. 2015;24:159-72. Medline:26028628 doi:10.1183/16000617.00000315

33 Pinnock H, Ostrem A, Rodriguez MR, Ryan D, Stallberg B, Thomas M, et al. Prioritising the respiratory research needs of primary care: the International Primary Care Respiratory Group (IPCRG) e-Delphi exercise. Prim Care Respir J. 2012;21:1927. Medline:22273628 doi:10.4104/pcrj.2012.00006 
34 Malhotra A, Schwartz AR, Schneider H, Owens RL, DeYoung P, Han MK, et al. Research Priorities in Pathophysiology for Sleep-disordered Breathing in Patients with Chronic Obstructive Pulmonary Disease. An Official American Thoracic Society Research Statement. Am J Respir Crit Care Med. 2018;197:289-99. Medline:29388824 doi:10.1164/rccm.201712-2510ST

35 Rudan I, Yoshida S, Chan KY, Cousens S, Sridhar D, Bahl R, et al. Setting health research priorities using the CHNRI method: I. Involving funders. J Glob Health. 2016;6:010301. Medline:26401269 doi:10.7189/jogh.06.010301

36 Yoshida S, Cousens S, Wazny K, Chan KY. Setting health research priorities using the CHNRI method: II. Involving researchers. J Glob Health. 2016;6:010302. Medline:27350870 doi:10.7189/jogh.06.010302

37 Yoshida S, Wazny K, Cousens S, Chan KY. Setting health research priorities using the CHNRI method: III. Involving stakeholders. J Glob Health. 2016;6:010303. Medline:27303649 doi:10.7189/jogh.06.010303

38 Rudan I. Setting health research priorities using the CHNRI method: IV. Key conceptual advances. J Glob Health. 2016;6:010501. Medline:27418959 doi:10.7189/jogh.06.010501

39 Rudan I, Yoshida S, Wazny K, Chan KY, Cousens S. Setting health research priorities using the CHNRI method: V. Quantitative properties of human collective knowledge. J Glob Health. 2016;6:010502. Medline:27350873 doi:10.7189/jogh.06.010502

40 Yoshida S, Rudan I, Cousens S. Setting health research priorities using the CHNRI method: VI. Quantitative properties of human collective opinion. J Glob Health. 2016;6:010503. Medline:27350874 doi:10.7189/jogh.06.010503

41 Rudan I, Gibson JL, Ameratunga S, El Arifeen S, Bhutta ZA, Black M, et al; Child Health and Nutrition Research Initiative. Setting priorities in global child health research investments: guidelines for implementation of CHNRI method. Croat Med J. 2008;49:720-33. Medline:19090596 doi:10.3325/cmj.2008.49.720

42 van Boven JF. Costs of case finding uncovered: time to revisit COPD's value pyramid? Thorax. 2019;74:727-9. Medline:31285362 doi:10.1136/thoraxjnl-2019-213440

43 Holgate ST. Priorities for respiratory research in the UK. Thorax. 2007;62:5-7. Medline:17189530 doi:10.1136/thx.2006.073882

44 Caron-Flinterman JF, Broerse JE, Teerling J, Bunders JF. Patients' priorities concerning health research: the case of asthma and COPD research in the Netherlands. Health Expect. 2005;8:253-63. Medline:16098155 doi:10.1111/j.1369-7625.2005.00337.x

45 Michalovic E, Sweet SN, Jensen D. Research and Healthcare Priorities of Individuals Living with COPD. COPD. 2021;18:13346. Medline:33779454 doi:10.1080/15412555.2021.1901271

46 López-Campos JL, Rodríguez DA, Quintana-Gallego E, Martínez-Llorens J, Hernández LC, Barreiro E. Ten Research Questions for Improving COPD Care in the Next Decade. COPD. 2019;16:311-20. Medline:31576763 doi:10.1080/15412555.20 19.1668919

47 Tabyshova A, Hurst JR, Soriano JB, Checkley W, Wan-Chun Huang E, Trofor AC, et al. Gaps in COPD guidelines of low- and middle-income countries: a systematic scoping review. Chest. 2021;159:575-84. Medline:33038390 doi:10.1016/j. chest.2020.09.260

48 Furman D, Campisi J, Verdin E, Carrera-Bastos P, Targ S, Franceschi C, et al. Chronic inflammation in the etiology of disease across the life span. Nat Med. 2019;25:1822-32. Medline:31806905 doi:10.1038/s41591-019-0675-0

49 Slavich GM. Psychoneuroimmunology of stress and mental health. In Harkness KL, Hayden EP (Eds.), The Oxford handbook of stress and mental health. New York: Oxford University Press; 2020.

50 Lu Y, Nyunt MSZ, Gwee X, Feng L, Feng L, Kua EH, et al. Life event stress and chronic obstructive pulmonary disease (COPD): Associations with mental well-being and quality of life in a population-based study. BMJ Open. 2012;2:e001674. Medline:23166130 doi:10.1136/bmjopen-2012-001674

51 Vardavas CI, Kyriakos CN, Fernández E, Bamidis P, Siddiqi K, Chavannes NH, et al. H2020 funding for respiratory research: scaling up for the prevention and treatment of lung diseases. Eur Respir J. 2019;54:1901417. Medline:31488589 doi:10.1183/13993003.01417-2019 